\title{
Transcriptional Analysis of Microcystis aeruginosa Co-Cultured with Algicidal Bacteria Brevibacillus laterosporus
}

\author{
Yulei Zhang ${ }^{1,2} \mathbb{D}$, Dong Chen ${ }^{1}$, Ning Zhang ${ }^{1,2}$, Feng Li $^{1,2}{ }^{\mathbb{D}}$, Xiaoxia Luo ${ }^{1}$, Qianru Li ${ }^{1}$, Changling Li ${ }^{1,2, *}$ \\ and Xianghu Huang 1,2,* \\ 1 Department of Aquaculture, Fisheries College, Guangdong Ocean University, Zhanjiang 524088, China; \\ zhangyl@gdou.edu.cn (Y.Z.); 18348445609@163.com (D.C.); zhangn@gdou.edu.cn (N.Z.); \\ lifeng2318@gdou.edu.cn (F.L.); xialemon@126.com (X.L.); liqianru01@163.com (Q.L.) \\ 2 Shenzhen Research Institute, Guangdong Ocean University, Shenzhen 518108, China \\ * Correspondence: licl@gdou.edu.cn (C.L.); huangxh@gdou.edu.cn (X.H.); \\ Tel.: +86-138-2252-1461 (C.L.); +86-138-0975-7646 (X.H.)
}

check for updates

Citation: Zhang, Y.; Chen, D.; Zhang, N.; Li, F.; Luo, X.; Li, Q.; Li, C.;

Huang, X. Transcriptional Analysis of Microcystis aeruginosa Co-Cultured with Algicidal Bacteria Brevibacillus laterosporus. Int. J. Environ. Res. Public Health 2021, 18, 8615. https:// doi.org/10.3390/ijerph18168615

Academic Editors: Alexandre Lamas, Patricia Regal and Nikolay Vassilev

Received: 16 June 2021

Accepted: 12 August 2021

Published: 15 August 2021

Publisher's Note: MDPI stays neutral with regard to jurisdictional claims in published maps and institutional affiliations.

Copyright: (c) 2021 by the authors. Licensee MDPI, Basel, Switzerland. This article is an open access article distributed under the terms and conditions of the Creative Commons Attribution (CC BY) license (https:/ / creativecommons.org/licenses/by/ $4.0 /)$.

\begin{abstract}
Harmful algal blooms caused huge ecological damage and economic losses around the world. Controlling algal blooms by algicidal bacteria is expected to be an effective biological control method. The current study investigated the molecular mechanism of harmful cyanobacteria disrupted by algicidal bacteria. Microcystis aeruginosa was co-cultured with Brevibacillus laterosporus Bl-zj, and RNA-seq based transcriptomic analysis was performed compared to M. aeruginosa, which was cultivated separately. A total of 1706 differentially expressed genes were identified, which were mainly involved in carbohydrate metabolism, energy metabolism and amino acid metabolism. In the co-cultured group, the expression of genes mainly enriched in photosynthesis and oxidative phosphorylation were significantly inhibited. However, the expression of the genes related to fatty acid synthesis increased. In addition, the expression of the antioxidant enzymes, such as 2-Cys peroxiredoxin, was increased. These results suggested that $B$. laterosporus could block the electron transport by attacking the PSI system and complex I of $M$. aeruginosa, affecting the energy acquisition and causing oxidative damage. This further led to the lipid peroxidation of the microalgal cell membrane, resulting in algal death. The transcriptional analysis of algicidal bacteria in the interaction process can be combined to explain the algicidal mechanism in the future.
\end{abstract}

Keywords: algicidal bacteria; Brevibacillus laterosporus; Microcystis aeruginosa; transcriptome; oxidative damage

\section{Introduction}

With the increase in water eutrophication, harmful algal blooms (HABs) have frequently occurred worldwide. In HABs, cyanobacteria are the most common group of microalgae, which can produce toxins [1-3]. Cyanobacterial blooms can cause serious water environmental problems, such as water oxygen deprivation, odorous substances and toxins, which are harmful to drinking water, aquatic animals, even threatening to human health [4-6]. Various methods have been proposed for removing or inhibiting cyanobacterial blooms, which include physical, chemical and biological methods. However, physical and chemical methods are expensive and cause secondary pollution, which is potentially harmful to aquatic ecosystems [7]. In contrast, biological methods are effective strategies to control HABs [8].

Algicidal bacteria are a group of bacteria that can kill or inhibit microalgae. At present, many algicidal bacteria, such as Bacillus [9], Chryseobacterium [10], Sulfitobacter [11], Hahella [12] and Stenotrophomonas [13], have been previously reported. There are two main mechanisms of algicidal bacteria: (1) direct attack: algicidal bacteria directly invade the microalgal cells; (2) indirect attack: algicidal bacteria inhibit microalgal growth by secreting extracellular active substances to lyse them or by competing with them for nutrients. The 
first study of algicidal bacteria was reported in Myxobacter that inhibited the growth of blue-green algae by secreting some cellulolytic enzymes and digesting the cell wall of the host [14]. Similarly, Furusawa et al. [15] found that Saprospira sp. SS98-5 exhibited an algicidal effect on Chaetoceros ceratosporum, using its filamentous cells to trap the diatom cells and dissolve their cytoderm, which were in contact with the bacteria. The specific or non-specific extracellular substances secreted by algicidal bacteria during their metabolism can destroy the structure of algal cells and lyse them. Extracellular active substances often act through various mechanisms to dissolve the cyanobacteria, including blocking electron transfer, reducing algal photosynthetic system activity, causing oxidative damage, inhibiting intracellular protein and carbohydrate synthesis and disrupting normal cell metabolism and cellular structure [16-18]. Yang et al. found that prodigiosin (PG) from Hahella sp. KA22 inhibited Microcystis aeruginosa by generating reactive oxygen species (ROS), which induce lipid peroxidation, disrupt the membrane system and obliterate the function of the photosystem [12]. In addition, many proteins of $M$. aeruginosa involved in important metabolic processes in response to PG stress were differentially expressed, which may trigger necrotic-like or apoptotic-like cell death with features similar to those in eukaryotes [12].

The algicidal mechanism has been extensively studied by measuring the physiological and biochemical characteristics of microalgae. A few studies have been conducted on the mechanism of algal-lysing at the molecular and protein levels. RNAseq and microarray analysis were determined to study the interactions between Roseobacters and dinoflagellates. The data suggested that at the early mutualistic phase of the symbiosis, polyhydroxyalkanoate (PHA) degradation might be the main carbon and energy source of Dinoroseobacter shibae, supplemented in the light by degradation of dimethylsulfoniopropionate (DMSP) and aerobic anoxygenic photosynthesis [19]. Proteomic analysis was applied to investigate the algicidal process of Trametes versicolor F21a on M. aeruginosa, and 30 fungal enzymes with endo- or exoglycosidase activities were significantly up-regulated, suggesting that these enzymes may degrade lipopolysaccharides, peptidoglycans and alginic acid of algal cells [20]. All these studies were analyzed from the algicidal bacteria or fungi, while the analysis of lysed microalgae at a molecular level may provide a new understanding of the algicidal mechanism.

Brevibacillus laterosporus is a Gram-positive bacterium that can produce antibacterial molecules and other secondary metabolites that have a wide range of biological activities [21]. In a previous study, the B. laterosporus strain Bl-zj was isolated from the intertidal soil of a park in Zhanjiang, which has been proved to have an algae-lytic effect on cyanobacteria, and the potential algicidal factors were screened by genome analysis [22,23].

The research on the mechanism of algicidal bacteria has been mainly conducted using various physiological and biochemical analyses; however, there is a scarcity of reports at the gene level, especially from the lysed microalgae. In this study, the algicidal bacteria B. laterosporus $\mathrm{Bl}-\mathrm{zj}$ and M. aeruginosa were co-cultured, and transcriptome analyses were performed to investigate the response of M. aeruginosa to algicidal bacteria.

\section{Materials and Methods}

\subsection{Cyanobacteria and Algicidal Bacterium}

M. aeruginosa FACHB 905 was purchased from the Freshwater Algae Culture Collection at the Institute of Hydrobiology, Wuhan, China. For inoculum preparation, it was cultured for $7 \mathrm{~d}$ to reach the exponential growth phase. It was cultivated in BG11 [24] medium at $28 \pm 1{ }^{\circ} \mathrm{C}$ under $50 \mu \mathrm{mol} \cdot \mathrm{m}^{-2} \cdot \mathrm{s}^{-1}$ with a photoperiod of $12: 12 \mathrm{~h}$ light: dark cycle. The concentration of $M$. aeruginosa was adjusted to $1 \times 10^{7}$ cells $/ \mathrm{mL}$ for further experiments.

B. laterosporus Bl-zj was isolated and preserved by the Laboratory of Algae Resource Development and Culture Environment Ecological Restoration of Guangdong Ocean University. It was cultured using Beef extract-peptone (BP) medium and incubated for $24 \mathrm{~h}$ at $28^{\circ} \mathrm{C}$ and $150 \mathrm{rpm}$ to reach the logarithmic growth phase. The concentration of $B$. laterosporus was adjusted to $1 \times 10^{7}$ cells $/ \mathrm{mL}$ for further experiments. 


\subsection{Measurement of the Algicidal Efficiency}

Equivalent volumes of microalga and bacterium $(10 \mathrm{~mL})$ were mixed, and total culture volume was added to $100 \mathrm{~mL}$ with BG11 medium (MB group). The control group was just $10 \mathrm{~mL}$ of $M$. aeruginosa added in BG11 medium to the total volume of $100 \mathrm{~mL}$ (BG11 group). Each group contained triplicate samples. The experiment lasted 4 days under the same condition as 2.1 when the MB group culture was turned yellow, and the microalgal biomass was at a low proportion.

Spectrophotometer with a $1 \mathrm{~cm}$ light path cuvette was used to measure and calculate chlorophyll $a$ concentration, according to the methods of Jeffrey and Humphrey [25]. In brief, the cultures of each group were harvested at 0, 1, 2, 3, $4 \mathrm{~d}$, and chlorophyll $a$ was extracted with $90 \%(v / v)$ acetone for $24 \mathrm{~h}$ in the dark. The absorbance of the supernatant at $630 \mathrm{~nm}, 647 \mathrm{~nm}$ and $664 \mathrm{~nm}$ was determined. The chlorophyll $a$ concentration was calculated according to the formula: $\mathrm{Chl} a(\mathrm{mg} / \mathrm{L})=\left(11.85 \times \mathrm{A}_{664}-1.54 \times \mathrm{A}_{647}-0.08 \times \mathrm{A}_{630}\right) \times \mathrm{V}_{1} / \mathrm{V}_{2}$, where $\mathrm{A}_{630}$, $A_{647}$ and $A_{664}$ represent absorbance, $V_{1}$ and $V_{2}$ are the volume of $90 \%(v / v)$ acetone $(m L)$ and cultures $(\mathrm{mL})$, respectively. The removal rate of microalgal cells $(\mathrm{R}, \%)$ was represented as the algicidal effect and calculated by the formula: $R=\left(C_{0}-C_{1}\right) / C_{0} \times 100 \%$, where $C_{0}$ and $C_{1}$ represent the chlorophyll $a$ concentration of BG11 group and MB group, respectively.

\subsection{Transcriptomic Samples Preparation}

The $M$. aeruginosa and B. laterosporus were co-cultured at a volume ratio of 1:1 (50 $\mathrm{mL} M$. aeruginosa, $50 \mathrm{~mL}$ B. laterosporus and $400 \mathrm{~mL}$ BG11 medium). M. aeruginosa was separately cultured as control (50 mL M. aeruginosa and $450 \mathrm{~mL}$ BG11 medium). Samples from the control group (named "CMA") and experimental group were collected on the second and fourth day (named "MB2" and "MB4", respectively), as the algicidal effect was obviously on the fourth day. The cocultures were centrifuged at $5000 \mathrm{rpm}, 4^{\circ} \mathrm{C}$ for $10 \mathrm{~min}$ to collect the pellets and then quickly frozen in liquid nitrogen and stored at $-80^{\circ} \mathrm{C}$. Each group contained triplicate samples.

\subsection{Transcriptomic Analysis}

Total RNA was extracted using RNAprep Pure Plant Plus Kit (Polysaccharides and Polyphenolics-rich, Tangen, China). Transcriptome sequencing (RNA-seq) was performed by Next-Generation Sequencing on the Illumina HiSeq 4000 platform. To obtain highquality clean data, the raw reads were filtered by removing adaptor sequences and lowquality reads ( $\mathrm{Q}$-value $\leq 20$ ). The clean reads were then mapped onto the M. aeruginosa PCC 7806SL complete genome (NCBI reference sequence, CP020771.1) using Bowtie2 program (http:/ / bowtie-bio.sourceforge.net/bowtie2/index.shtml (version 2.4.1, accessed on 28 February 2020)).

The differences in gene expression were analyzed by HTSeq (version0.6.1p2) for the screening of differentially expressed genes (DEGs) and principal components analysis. The read count mapped to each gene was calculated as the original expression of the gene and the FPKM (Fragments Per Kilo bases per Million fragments) was used to normalize the expression levels. The DEGs screening conditions were as follows: $\log _{2} \mid$ fold change $\mid>1$, $p$-value $<0.05$. DEGs were further annotated to Gene Ontology (GO) database and Kyoto Encyclopedia of Genes and Genomes (KEGG) database.

\subsection{Quantitative Real-Time PCR Validation}

Quantitative PCR (qPCR) was performed to verify the results of mRNA-seq analysis. The cDNA template was synthesized by reverse transcription using a kit (HiScript ${ }^{\mathrm{R}}$ III RT SuperMix for qPCR (+gDNA wiper), Vazyme Biotechnology Co., LTD., Nanjing, China). The qPCR was performed using SYBR green (AceQ Universal SYBR qPCR Master Mix, Vazyme Biotechnology Co., LTD., Nanjing, China) on a 7500 Fast Real-time PCR System (Applied Biosystems, Foster, CA, USA). The primers were designed according to the gene sequences from M. aeruginosa PCC 7806SL complete genome, with 16S rRNA [26] as the 
reference gene and synthesized by GENEWIZ (Suzhou, China) (the primers were shown in Supplementary Table S1).

\section{Results}

\subsection{The Algicidal Efficiency}

Compared with the control group (BG11), the chlorophyll $a$ concentration of $M$. aeruginosa significantly decreased with the addition of $\mathrm{B}$. laterosporus from the second day (Figure 1). The removal rates of M. aeruginosa in the MB group were $34.39 \%, 72.36 \%$ and $92.30 \%$ on the second, third and fourth days, respectively, showing an increasing algicidal effect over time.

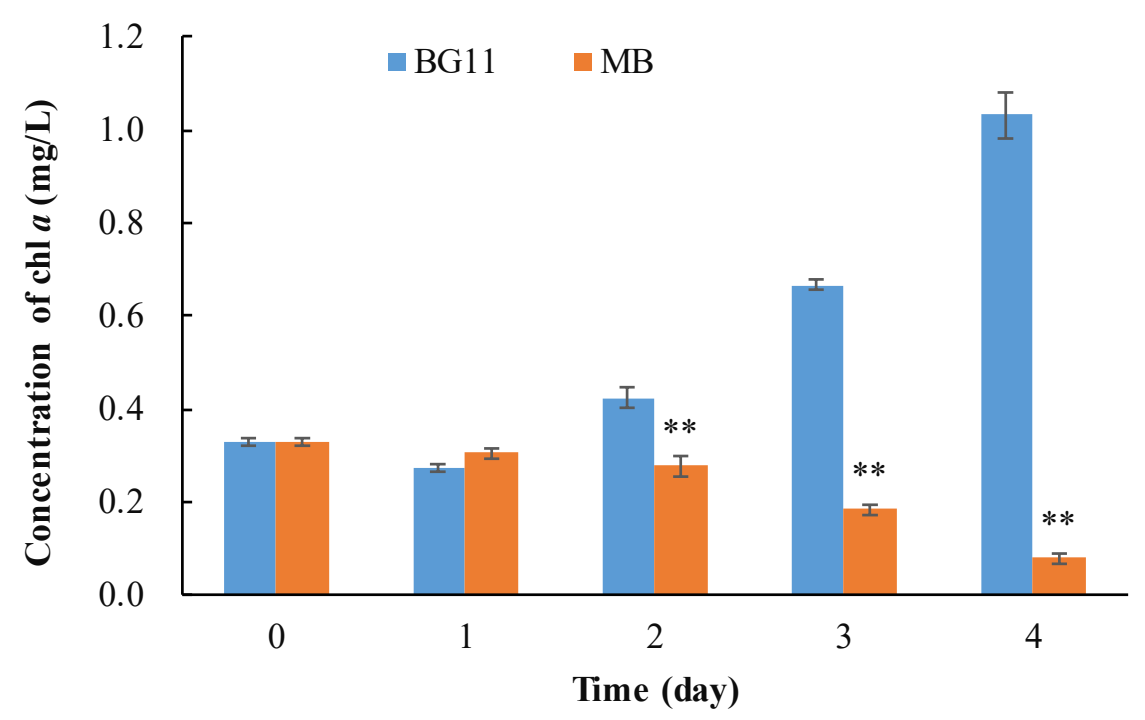

Figure 1. Algicidal effect of B. laterosporus on M. aeruginosa. (** Represents significant differences to control group at the 0.01 probability level.)

\subsection{Illumina Sequencing Assembly Data Quality Analysis}

Nine cDNA libraries from three groups were sequenced to study the transcriptomes of $M$. aeruginosa under the effect of B. laterosporus (Table 1). An average of 9,728,733,733 bp raw data and $64,858,225$ raw reads were obtained from each sample. After filtering the adaptors and low-quality sequences from raw data, an average of 7,800,583,533 bp clean data and 52,003,890 clean reads were screened. After mapping to the reference genome, an average of 37,785,780 mapped reads were obtained. All Q20 and Q30 values of the read sequences in the samples exceeded $96.94 \%$ and $92.9 \%$, respectively.

Table 1. Summary of transcriptome sequencing data of M. aeruginosa under B. laterosporus.

\begin{tabular}{|c|c|c|c|c|c|c|c|}
\hline Sample & Raw Data (bp) & $\begin{array}{c}\text { Raw Reads } \\
\text { No. }\end{array}$ & $\begin{array}{l}\text { Clean Data } \\
\text { (bp) }\end{array}$ & $\begin{array}{c}\text { Clean Reads } \\
\text { No. }\end{array}$ & $\begin{array}{c}\text { Mapped Reads } \\
\text { No. }\end{array}$ & Q20 (\%) & Q30 (\%) \\
\hline CMA1 & $9,468,720,000$ & $63,124,800$ & $8,089,035,900$ & $53,926,906$ & $52,199,272$ & 97.86 & 94.09 \\
\hline CMA2 & $9,206,526,300$ & $61,376,842$ & $7,486,911,900$ & $49,912,746$ & $48,062,591$ & 97.93 & 94.40 \\
\hline CMA3 & $8,543,553,900$ & $56,957,026$ & $7,371,049,200$ & $49,140,328$ & $47,594,707$ & 98.05 & 94.50 \\
\hline MB2_1 & $11,260,710,900$ & $75,071,406$ & $9,110,216,700$ & $60,734,778$ & $43,388,415$ & 97.73 & 94.22 \\
\hline MB2_2 & $9,961,599,300$ & $66,410,662$ & $7,954,650,900$ & $53,031,006$ & $39,017,497$ & 97.62 & 93.88 \\
\hline MB2_3 & $9,498,113,100$ & $63,320,754$ & $7,660,962,000$ & $51,073,080$ & $36,364,637$ & 97.65 & 93.90 \\
\hline MB4_1 & $10,501,108,500$ & $70,007,390$ & $8,108,475,900$ & $54,056,506$ & $37,308,387$ & 97.72 & 94.15 \\
\hline MB4_2 & $9,475,501,500$ & $63,170,010$ & $7,402,896,600$ & $49,352,644$ & $4,777,919$ & 97.29 & 93.41 \\
\hline MB4_3 & $9,642,770,100$ & $64,285,134$ & $7,021,052,700$ & $46,807,018$ & $31,358,598$ & 96.94 & 92.90 \\
\hline Average & $9,728,733,733$ & $64,858,225$ & $7,800,583,533$ & $52,003,890$ & $37,785,780$ & 97.64 & 93.94 \\
\hline
\end{tabular}


Principal component analysis (PCA) was performed for each sample according to the expression to observe similarities between sample groups (Figure 2). The three replicates of each group (CMA, MB2 and MB4) were gathered with close distance, but different treatment groups separated from others at a long distance, indicating good repeatability data.

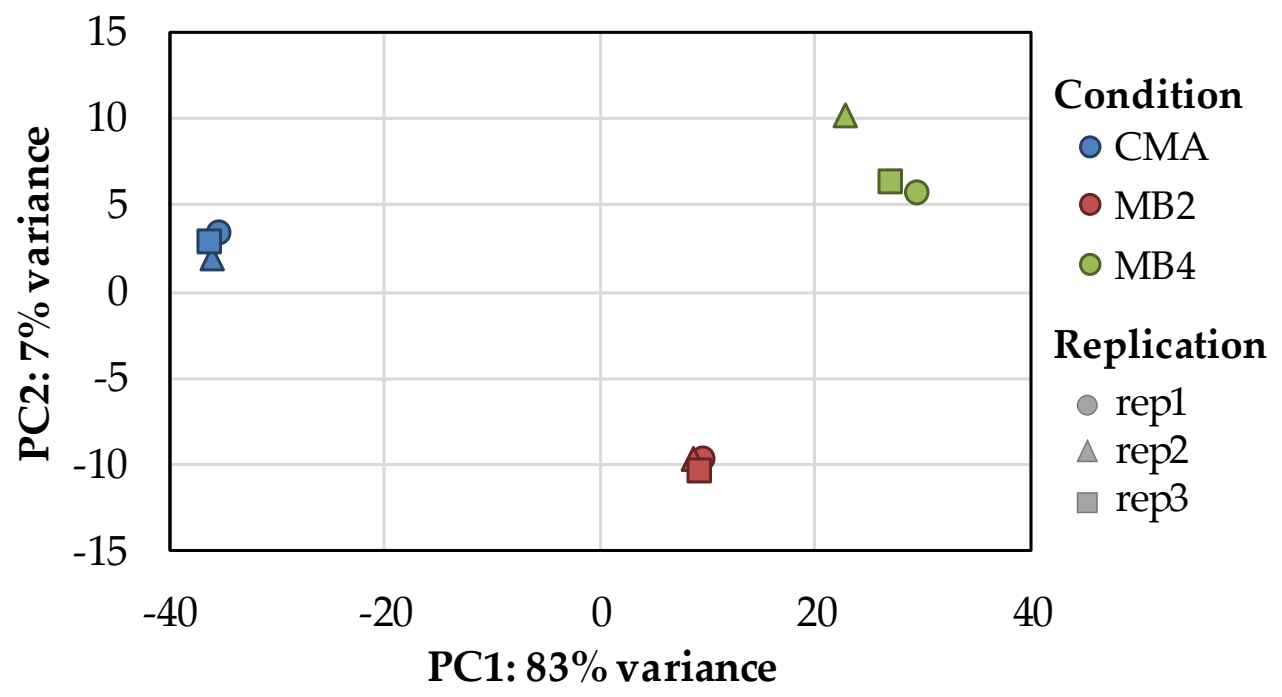

Figure 2. The principal component analysis (PCA) was performed for each sample. (The blue, red and green labels represent the treatment group of CMA, MB2 and MB4, respectively. The circle, triangle and square labels represent replication 1, replication 2 and replication 3 samples in each group, respectively.)

\subsection{Identification of DEGs}

In this study, transcriptomic analysis was used to investigate the impact of algicidal bacteria B. laterosporus Bl-zj on M. aeruginosa metabolism at the molecular level. Results showed that a total of 1324 DEGs were found in the MB2 group compared to CMA, of which 612 were up-regulated and 712 were down-regulated (Figure 3). There were 1289 DEGs in the MB4 group compared to CMA, of which 450 were up-regulated and 839 were down-regulated. A total of 1706 DEGs were identified; among them, there were 377 up-regulated and 528 down-regulated DEGs both in MB2 vs. CMA comparison and MB4 vs. CMA comparison.

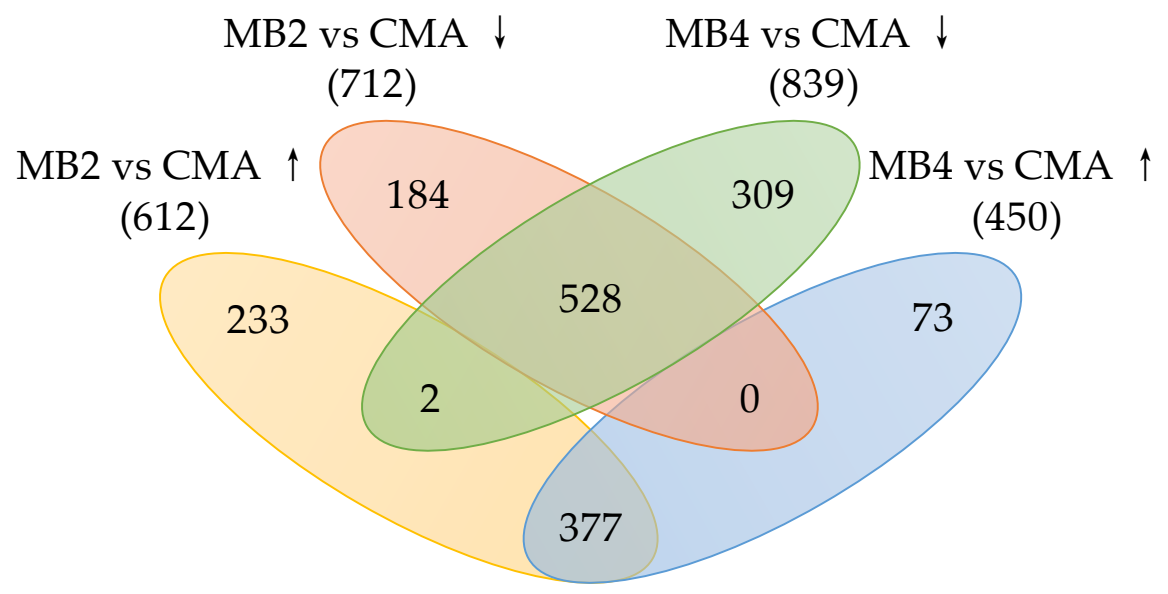

Figure 3. The number of differentially expressed genes (DEGs) at CM2 and CM4 groups compared with CMA group. (“ $\uparrow "$ and " $\downarrow$ " represent up-regulated and down-regulated genes, respectively.) 


\subsection{Functional Classification of the DEGs by GO and KEGG Pathway Analysis}

The GO and KEGG pathways were used to analyze the DEGs and determine their main biological functions to explore the molecular changes in M. aeruginosa under the effect of B. laterosporus Bl-zj.

The top 10 most significant enriched genes in three GO categories were summarized (Figure 4). In the cellular component category, the GO terms mainly enriched in the MB2 vs. CMA comparison included "photosystem I", "cell part" and "cytoplasm". The "intracellular part", "intracellular" and "cell part" were mainly enriched in the MB4 vs. CMA comparison. Similarly, in the molecular function category, "ion binding", "quinone binding" and "small molecule binding" were significantly enriched in the MB2 vs. CMA comparison. The "ion binding", "small molecule binding" and "oxidoreductase activity" were significantly enriched in the MB4 vs. CMA comparison. Further, in the biological process, "prophyrin-containing compound biosynthetic process", "oxidative phosphorylation" and "prophyrin-containing compound metabolic process" were significantly enriched in the MB2 vs. CMA comparison. The "photosynthesis", "pigment biosynthetic process" and "oxidation-reduction process" were significantly enriched in the MB4 vs. CMA comparison.

The biological functions associated with DEGs were further analyzed using the KEGG database. The up-regulated and down-regulated genes were involved in carbohydrate metabolism, amino acid metabolism, metabolism of cofactors and vitamins and energy metabolism in MB2 vs. CMA comparison and MB4 vs. CMA comparison (Table 2). In addition, the genes of lipid metabolism and translation were mainly up-regulated and the genes of energy metabolism, replication and repair and membrane transport were mainly down-regulated.

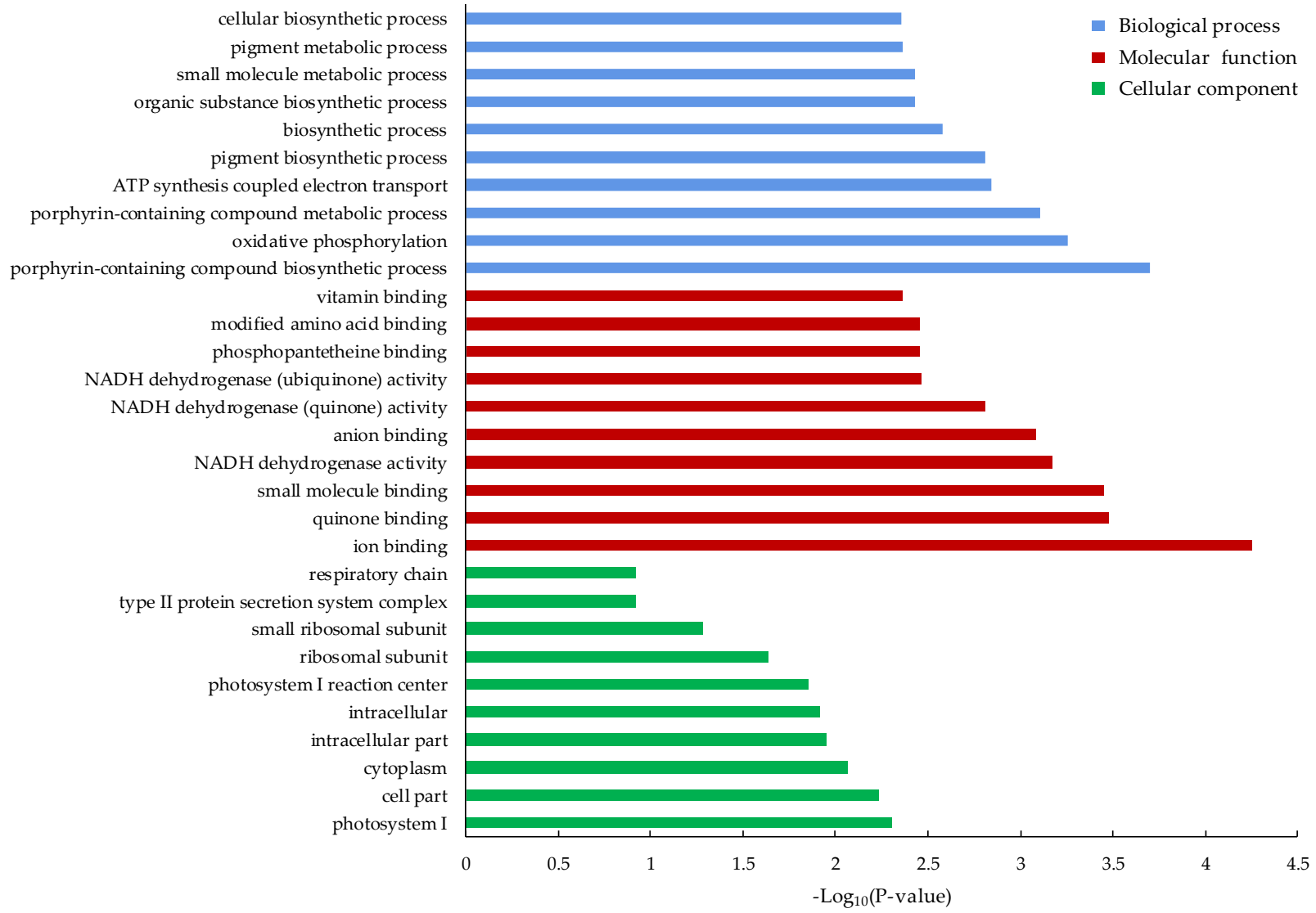

(a)

Figure 4. Cont. 


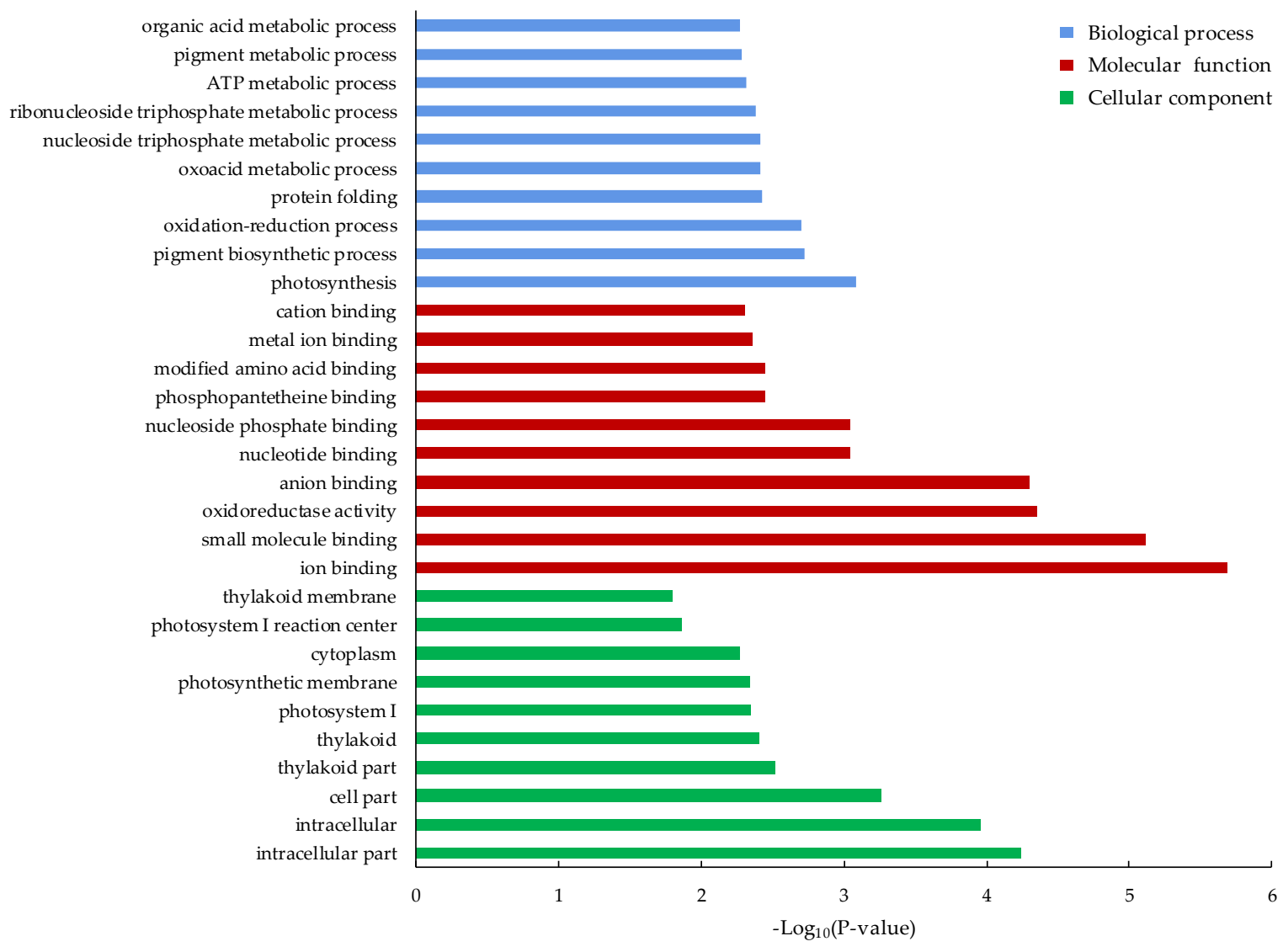

(b)

Figure 4. Gene ontology (GO) enrichment analysis of the differently expressed genes at two groups compared with CMA group. (a) MB2 vs. CMA comparison; (b) MB4 vs. CMA comparison.

Table 2. Distribution of up- and down-regulated genes in KEGG pathways.

\begin{tabular}{|c|c|c|c|c|}
\hline \multirow{2}{*}{ KEGG Pathways (Level 2) } & \multicolumn{2}{|c|}{ MB2 vs. CMA } & \multicolumn{2}{|c|}{ MB4 vs. CMA } \\
\hline & $\mathrm{Up}$ & Down & Up & Down \\
\hline Amino acid metabolism & 42 & 25 & 39 & 38 \\
\hline Carbohydrate metabolism & 59 & 40 & 52 & 60 \\
\hline Energy metabolism & 31 & 44 & 27 & 52 \\
\hline Metabolism of cofactors and vitamins & 33 & 15 & 28 & 23 \\
\hline Lipid metabolism & 15 & 6 & 16 & 11 \\
\hline Translation & 16 & 3 & 14 & 2 \\
\hline Metabolism of other amino acids & 9 & 4 & 9 & 4 \\
\hline Biosynthesis of other secondary metabolites & 7 & 4 & 9 & 5 \\
\hline Nucleotide metabolism & 12 & 11 & 7 & 15 \\
\hline Metabolism of terpenoids and polyketides & 8 & 7 & 7 & 4 \\
\hline Replication and repair & 6 & 10 & 5 & 14 \\
\hline Membrane transport & 4 & 8 & 2 & 9 \\
\hline Xenobiotics biodegradation and metabolism & 5 & 2 & 3 & 3 \\
\hline Transcription & 2 & 1 & 2 & 1 \\
\hline Signal transduction & 1 & 4 & 1 & 3 \\
\hline Immune system & 0 & 2 & 0 & 2 \\
\hline Glycan biosynthesis and metabolism & 2 & 2 & 1 & 4 \\
\hline Folding, sorting and degradation & 8 & 3 & 5 & 8 \\
\hline Cell growth and death & 1 & 1 & 1 & 1 \\
\hline Cellular community-prokaryotes & 1 & 7 & 1 & 8 \\
\hline Environmental adaptation & - & - & 0 & 1 \\
\hline
\end{tabular}




\subsection{Algicidal-Related Gene and Pathway Analysis}

The expression of genes related to photosynthesis, oxidative phosphorylation and fatty acid synthesis in $M$. aeruginosa were significantly changed under the effect of $B$. laterosporus Bl-zj (Table 3). There were nine photosynthesis-related genes that were down-regulated on the second and fourth days, compared to the CMA group, among which seven genes were related to photosystem I (PSI). Nine genes related to oxidative phosphorylation were downregulated both on the second and fourth days, compared to the control. The expression of ND5 and Ndufs 8 were most significantly decreased with $\log _{2}$ (fold changes) of 3.38 and 3.43 on the fourth day compared to the control, respectively. In addition, the expression of two genes (FabF and FabG1) involved in fatty acid synthesis increased on the second day compared to the control, while YOXD was increased on the fourth day compared to the control. Similarly, three genes (FabH, FabG2 and FabZ) were up-regulated on the second and fourth days compared to the control.

Table 3. Potential candidate genes related to the response to B. laterosporus Bl-zj treatment (FC = fold change).

\begin{tabular}{|c|c|c|c|c|c|c|}
\hline \multirow{2}{*}{ Gene ID } & \multirow{2}{*}{ Symbol } & \multicolumn{2}{|c|}{ MB2 vs. CMA } & \multicolumn{2}{|c|}{ MB4 vs. CMA } & \multirow{2}{*}{ Annotation } \\
\hline & & $\log _{2} \mathrm{FC}$ & $p$-Value & $\log _{2} \mathrm{FC}$ & $p$-Value & \\
\hline \multicolumn{7}{|c|}{ Photosynthesis } \\
\hline RS02885 & PsaA & -1.02 & $1.66 \times 10^{-3}$ & -1.29 & $1.35 \times 10^{-3}$ & photosystem I core protein psaA \\
\hline RS02890 & PsaB & -1.34 & $2.02 \times 10^{-5}$ & -1.73 & $8.05 \times 10^{-6}$ & photosystem I core protein psaB \\
\hline RS22460 & PsaC & -2.5 & $1.13 \times 10^{-15}$ & -3.97 & $3.25 \times 10^{-19}$ & photosystem I subunit VII \\
\hline RS24715 & PsaD & -2.93 & $2.42 \times 10^{-23}$ & -4.04 & $6.13 \times 10^{-23}$ & photosystem I reaction center subunit II \\
\hline RS04855 & PsaE & -1.66 & $6.27 \times 10^{-13}$ & -1.97 & $6.76 \times 10^{-7}$ & photosystem I reaction center subunit IV \\
\hline RS02775 & PsaF & -1.61 & $3.01 \times 10^{-13}$ & -1.96 & $8.76 \times 10^{-8}$ & photosystem I subunit III \\
\hline RS25020 & PsaL & -3.61 & $1.36 \times 10^{-53}$ & -3.71 & $3.78 \times 10^{-19}$ & photosystem I reaction center subunit XI \\
\hline RS13445 & PC & -4.00 & $8.78 \times 10^{-65}$ & -3.40 & $3.01 \times 10^{-22}$ & plastocyanin \\
\hline RS12310 & FER & -3.24 & $4.87 \times 10^{-29}$ & -2.88 & $8.76 \times 10^{-13}$ & ferredoxin \\
\hline RS15355 & Psb28 & -1.12 & $2.85 \times 10^{-2}$ & - & - & photosystem II reaction center protein Psb28 \\
\hline RS02780 & PsaJ & -1.61 & $4.15 \times 10^{-6}$ & - & - & photosystem I reaction center subunit IX \\
\hline RS15435 & UCRI & - & - & -1.6 & $9.44 \times 10^{-5}$ & cytochrome b6-f complex iron-sulfur subunit 1 \\
\hline RS16845 & ISIA & 3.94 & $1.97 \times 10^{-61}$ & 3.36 & $6.36 \times 10^{-21}$ & iron stress-induced chlorophyll-binding protein \\
\hline RS13450 & CYC6 & 5.63 & $2.69 \times 10^{-17}$ & 4.63 & $2.31 \times 10^{-4}$ & cytochrome c6 \\
\hline RS12320 & PsbU & - & - & 1.17 & $2.53 \times 10^{-3}$ & photosystem II $12 \mathrm{kDa}$ extrinsic protein \\
\hline RS16660 & PsbJ & 1.08 & $1.00 \times 10^{-4}$ & - & - & photosystem II reaction center protein J \\
\hline \multicolumn{7}{|c|}{ Oxidative phosphorylation } \\
\hline RS07425 & ND1 & -1.38 & $7.03 \times 10^{-9}$ & -1.77 & $2.21 \times 10^{-4}$ & NAD(P)H-quinone oxidoreductase subunit 1 \\
\hline RS05160 & ND2 & -1.09 & $3.98 \times 10^{-7}$ & -1.72 & $5.03 \times 10^{-6}$ & $\mathrm{NAD}(\mathrm{P}) \mathrm{H}$-quinone oxidoreductase subunit 2 \\
\hline RS13045 & ND4 & -2.57 & $3.58 \times 10^{-10}$ & -2.61 & $1.02 \times 10^{-5}$ & $\begin{array}{c}\text { proton-translocating NADH-quinone oxidoreductase, chain } \\
\text { M family protein }\end{array}$ \\
\hline RS13470 & ND5 & -2.38 & $1.77 \times 10^{-16}$ & -3.38 & $1.70 \times 10^{-7}$ & NAD(P)H dehydrogenase, subunit NdhF3 family protein \\
\hline RS08000 & Ndufs1 & -1.39 & $4.18 \times 10^{-6}$ & -1.89 & $2.86 \times 10^{-6}$ & $2 \mathrm{Fe}-2 \mathrm{~S}$ iron-sulfur cluster binding domain protein \\
\hline RS07420 & Ndufs8 & -2.64 & $3.79 \times 10^{-14}$ & -3.43 & $6.95 \times 10^{-15}$ & NADH-plastoquinone oxidoreductase, I subunit \\
\hline RS23775 & COX10 & -1.35 & $6.50 \times 10^{-9}$ & -2.27 & $2.37 \times 10^{-11}$ & protoheme IX farnesyltransferase \\
\hline RS09740 & ATPF1D & -1.39 & $1.65 \times 10^{-4}$ & -2.01 & $1.96 \times 10^{-3}$ & ATP synthase F1, delta subunit \\
\hline RS09735 & ATPF0B & -1.57 & $1.47 \times 10^{-6}$ & -2.33 & $1.05 \times 10^{-7}$ & ATP synthase F0, B subunit \\
\hline RS24375 & ND3 & -2.33 & $1.35 \times 10^{-3}$ & - & - & NADH dehydrogenase subunit A \\
\hline RS05535 & Ndufv2 & -1.12 & $1.16 \times 10^{-6}$ & - & - & respiratory-chain NADH dehydrogenase $24 \mathrm{kDa}$ subunit \\
\hline RS23755 & $\mathrm{COX} 3$ & - & - & -1.64 & $9.83 \times 10^{-4}$ & cytochrome c oxidase subunit III \\
\hline RS09750 & ATPG & - & - & -1.5 & $1.37 \times 10^{-3}$ & ATP synthase gamma chain \\
\hline RS12325 & NADB & 1.5 & $3.14 \times 10^{-7}$ & - & - & L-aspartate oxidase \\
\hline RS23760 & COX1 & 1.37 & $7.16 \times 10^{-9}$ & - & - & cytochrome c oxidase, subunit I \\
\hline RS23765 & $\mathrm{COX} 2$ & 1.1 & $2.64 \times 10^{-7}$ & - & - & cytochrome c oxidase, subunit II \\
\hline RS24380 & Ndufs7 & - & - & 1.45 & $9.08 \times 10^{-5}$ & NAD(P)H-quinone oxidoreductase subunit K 1 \\
\hline RS13440 & DHSA & - & - & 1.53 & $9.29 \times 10^{-5}$ & $\begin{array}{l}\text { succinate dehydrogenase/fumarate reductase, flavoprotein } \\
\text { subunit }\end{array}$ \\
\hline \multicolumn{7}{|c|}{ Fatty acid biosynthesis } \\
\hline RS04585 & FabH & 1.88 & $3.38 \times 10^{-11}$ & 1.97 & $1.56 \times 10^{-2}$ & 3-oxoacyl-[acyl-carrier-protein] synthase III \\
\hline RS18070 & FabG2 & 1.31 & $2.24 \times 10^{-9}$ & 1.33 & $1.12 \times 10^{-2}$ & PHA-specific acetoacetyl-CoA reductase \\
\hline RS08035 & FabZ & 2.42 & $4.01 \times 10^{-13}$ & 2.51 & $6.76 \times 10^{-5}$ & beta-hydroxyacyl-(acyl-carrier-protein) dehydratase \\
\hline RS22350 & FabF & 1.32 & $6.66 \times 10^{-4}$ & - & - & beta-ketoacyl synthase, C-terminal domain protein \\
\hline RS12750 & FabG1 & 1.16 & $9.88 \times 10^{-5}$ & - & - & 3-oxoacyl-[acyl-carrier-protein] reductase \\
\hline RS05845 & YOXD & - & - & 2.26 & $3.16 \times 10^{-9}$ & short chain dehydrogenase family protein \\
\hline RS23000RS09855 & AAE16 & -2.73 & $8.26 \times 10^{-10}$ & -4.54 & $3.16 \times 10^{-11}$ & AMP-binding enzyme family protein \\
\hline KSZSUUUKSUYOJJ & FabI & - & - & -1.79 & $4.63 \times 10^{-3}$ & enoyl-[acyl-carrier-protein] reductase [NADH] \\
\hline \multicolumn{7}{|c|}{ Antioxidase } \\
\hline RS02415 & Y755 & 1.21 & $2.98 \times 10^{-8}$ & 2.52 & $4.86 \times 10^{-12}$ & 2-Cys peroxiredoxin BAS1 \\
\hline
\end{tabular}




\subsection{Quantitative Real-Time PCR Validation}

To confirm the RNA-seq results, seven genes were selected for further qRT-PCR analysis. Figure $5 \mathrm{a}, \mathrm{b}$ shows the expression levels of each gene on the second and fourth days, respectively. Although the fold changes were different, the expression trends of these seven genes were consistent in both RNA-seq and qRT-PCR results.

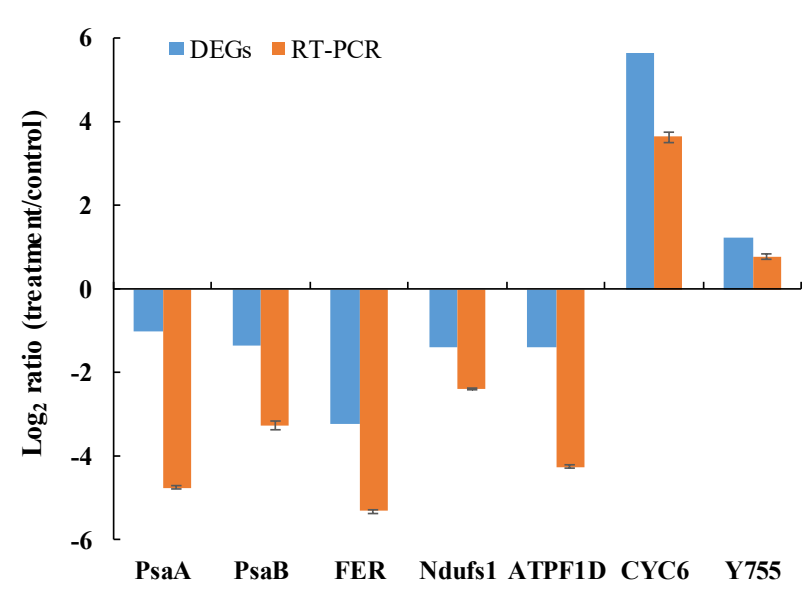

(a)

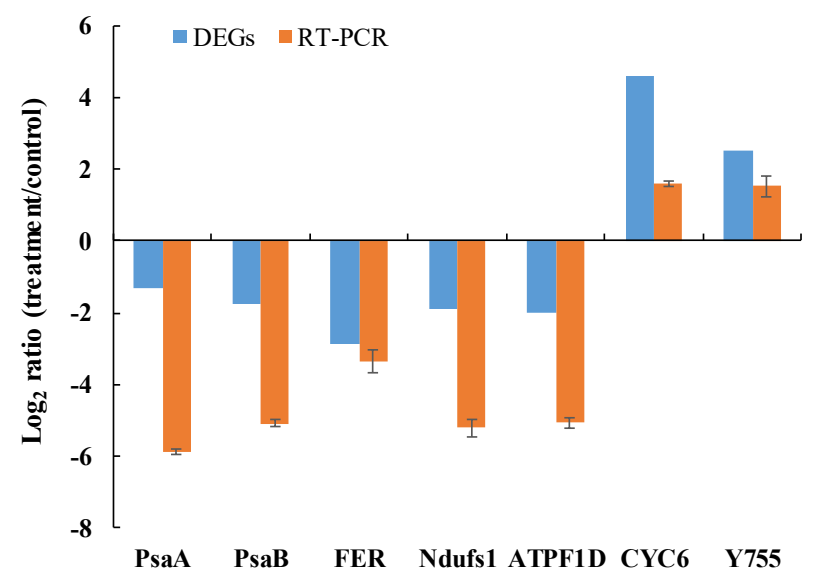

(b)

Figure 5. Comparison of the expressions of 7 differently expressed genes determined by Illumina HiSeq 2500 sequencing and qRT-PCR. (a) MB2 vs. CMA comparison (b) MB4 vs. CMA comparison.

\section{Discussion}

In the present study, second- and fourth-day transcriptomes of M. aeruginosa in coculture with B. laterosporus Bl-zj were obtained and compared using next-generation sequencing technology. We found that several pathways significantly changed, which were probably the critical factors as B. laterosporus attacked the cells of $M$. aeruginosa.

Photosynthesis is a critical cellular process that determines the growth of microalgae. In this process, light energy is captured and used to synthesize carbohydrates and produce oxygen while consuming carbon dioxide. Transcriptomic analysis showed that PSI was seriously affected by $B$. laterosporus Bl-zj. The principal subunits of the reaction center (PsaA and $\mathrm{PsaB}$ ) and other subunits (PsaC, PsaE, PsaD, PsaF and PsaL) were significantly downregulated on the second day and continuously inhibited on the fourth day (Table 3). PSI is a supercomplex of a reaction center and light-harvesting complexes [27]. PsaA and PsaB are the central core proteins that bonded all cofactors of the electron transport chain except the cluster of ferredoxins and harbor the electron transport chain [28]. PsaC can bind to the terminal electron acceptor and participate in the transfer of electrons to ferredoxin [29]. PsaE was responsible for the dissociation of ferredoxin from the PSI complex and involved in the cyclic electron flow that occurred around the PSI complex [30]. PsaD has been regarded as a key subunit in the assembly, stability and functionality of PSI [31]. PsaF has been found to be related to efficient electron transfer from both plastocyanin and cytochrome c6 to PSI [32]. It has been reported that PsaL was critical for energy transfer from phosphorylated LHCII to the PSI reaction center [33]. In this study, it was observed that Psb28 significantly declined after 2 days compared to the control. Psb28 was the photosystem II reaction center protein, which plays an important role in PSII repair [34]. In addition, plastocyanin and ferredoxin significantly decreased after the second and fourth days compared to the control. Plastocyanin and ferredoxin are small soluble copper protein and iron-sulfur protein, respectively, which are important PSI turnover products induced by light energy involved in electron transfer from plastocyanin to ferredoxin [28,35]. These results showed that under the action of B. laterosporus Bl-zj photosynthesis, especially the PSI of $M$. aeruginosa, is significantly inhibited. The reduction of photosynthesis-related 
gene transcripts impeded electron transport, resulting in the loss of equivalent products necessary for the process of carbon assimilation.

Oxidative phosphorylation is the main energy source of aerobic cells and the main pathway of ATP production [36]. It is an enzymatic process in which ATP is formed when electrons are transferred from substrate to oxygen and ADP is phosphorylated. Transcriptomic analysis showed that the genes related to oxidative phosphorylation significantly decreased after the second and fourth days, relative to the control (Table 3). NADH: ubiquinone oxidoreductase (Complex I) is the first and most complex enzyme required in this process. It connected NADH and ubiquinone electron transfer to proton transmembrane transport, which helped to generate proton power, which was necessary for ATP synthesis. Further, ND1, ND2, ND4, ND5, Ndufs1, Ndufs8 were the core subunits of Complex I [37]. In Chlamydomonas, the loss of ND1 or ND5 subunit prevented the assembly of whole mitochondrial Complex I, whereas the loss of ND4 led to the formation of a subcomplex of $650 \mathrm{kDa}$ present in a reduced amount $[38,39]$. In addition, COX10 related to cytochrome c oxidase (Complex IV) and $\mathrm{ATPF}_{0} \mathrm{~B}, \mathrm{ATPF}_{1} \mathrm{D}$ related ATP synthase significantly decreased after the second and fourth days compared to the control group. Complex IV catalyzes the last step of the mitochondrial electron transfer chain and is considered one of the main regulatory sites of oxidative phosphorylation [40]. COX10 participates in the biosynthesis of heme a, a key cofactor of cytochrome c oxidase [41]. ATP synthase can synthesize cellular ATP from ADP and inorganic phosphate. It is comprised of two parts, an ATP-driven motor $\mathrm{F}_{1}$ and a proton-driven $\mathrm{F}_{0}$ motor [42].

The down-regulation of oxidative phosphorylation expression led to a reduction in the transmembrane gradient and ATP synthesis, suggesting that B. laterosporus Bl-zj decreased the amount of cellular ATP in M. aeruginosa cells. Moreover, the absolute value of $\log _{2}$ (fold changes) of MB4 vs. CMA comparison were almost larger than that of MB2 vs. CMA comparison. This indicated that oxidative phosphorylation of M. aeruginosa was continuously inhibited. Further, the ATP synthesis decreased, and the cyanobacteria cells could not obtain enough energy to survive. In addition, the electron transport chain and oxidative phosphorylation are critical cellular processes that sustain life, and the absence of either can affect cellular respiration and even lead to cell death [43]. The decrease of electron transport chain and oxidative phosphorylation genes expression could inhibit the algal growth and even cause death.

The response and adaptability of microalgae to environmental changes were closely related to numerous changes in the composition of lipids in cells and the ability to synthesize a series of special lipids [44]. Under stress, some algal cells cannot reproduce but accumulate lipids instead $[45,46]$. Many deep-sea bacteria adapted to the deep-sea environment by synthesizing more unsaturated lipids [47]. In this study, the expression of fatty acid biosynthesis-related genes FabH and FabZ were up-regulated in both MB2 and MB4 groups compared to control, while FabF was up-regulated only in MB2 vs. CMA comparison (Table 3). FabF catalyzed the elongation of fatty acyl chains and NADPH-specific reduction of long-chain $\beta$-ketoacyl derivatives [48]. FabH was considered to catalyze the first elongation reaction (Claisen condensation) of type II fatty acid synthesis, resulting in the production of short-chain fatty acid primers [49]. FabZ showed ubiquitous distribution in type II fatty acid synthase, and the dehydratase efficiently catalyzed the dehydration of short and long-chain saturated and unsaturated beta-hydroxyacyl-ACPs [50]. The upregulated expression of fatty acid synthesis genes revealed that $M$. aeruginosa was under stress. When M. aeruginosa was under the stress of pyrogallol, a compound with strong inhibition on M. aeruginosa, the expression of FabZ was also increased [51]. Moreover, the expression of gene Y755 was up-regulated. 2-Cys peroxiredoxin was a subclass of peroxiredoxin proteins (Prx-s), and Prx-s were a ubiquitous family of antioxidant enzymes and important components of the cellular antioxidant defense system as well as redox homeostasis [52]. These suggested that membranes may undergo oxidative damage. In order to maintain the normal function of membranes, more unsaturated fatty acids need to be integrated into membranes. 


\section{Conclusions}

In the present study, we provide the molecular responses of $M$. aeruginosa to B. laterosporus treatment. The $B$. laterosporus treatment led to a suppression of photosynthesis and oxidative phosphorylation of M. aeruginosa. We also found that the expression of the genes related to fatty acid synthesis increased. In addition, the expression of the antioxidant enzymes, such as 2-Cys peroxiredoxin, was increased. The inhibition of photosynthesis and oxidative phosphorylation of $M$. aeruginosa could block electron transport and affect energy acquisition. The increase in fatty acid synthesis-related genes and 2-Cys peroxiredoxin indicated that cyanobacteria exhibited oxidative damage, causing algal cell membrane lipid peroxidation. These changes inhibited cyanobacteria growth, leading to algae death.

Supplementary Materials: The following are available online at https:/ /www.mdpi.com/article/10 .3390/ijerph18168615/s1, Table S1: PCR primer pairs used in this study.

Author Contributions: Conceptualization, Y.Z. and D.C.; Formal analysis, Y.Z., D.C., N.Z., F.L. and Q.L.; Funding acquisition, Y.Z., X.L., C.L. and X.H.; Writing—original draft, D.C.; Writing—review and editing, Y.Z., C.L. and X.H. All authors have read and agreed to the published version of the manuscript.

Funding: This research was funded by the National Key R \& D Plan "Blue Granary Science and Technology Innovation" (SQ2020YFD090053-05); the Guangdong Provincial Key Area Research and Development Plan Projects (2020B0202010009); the Guangdong South China Sea Key Laboratory of Aquaculture for Aquatic Economic Animals, Guangdong Ocean University (KFKT2019YB09); and the Natural Science Foundation of Guangdong Province (2018A030313212).

Institutional Review Board Statement: Not applicable.

Informed Consent Statement: Not applicable.

Conflicts of Interest: The authors declare no conflict of interest.

\section{References}

1. Watanabe, M.F.; Oishi, S.; Watanabe, Y.; Watanabe, M. Strong Probability Of Lethal Toxicity In The Blue-Green Alga Microcystis Viridis Lemmermann1. J. Phycol. 1986, 22, 552-556. [CrossRef]

2. Sivonen, K.; Carmichael, W.W.; Namikoshi, M.; Rinehart, K.L.; Dahlem, A.M.; Niemelä, S.I. Isolation and characterization of hepatotoxic microcystin homologs from the filamentous freshwater cyanobacterium Nostoc sp. strain 152. Appl. Environ. Microbiol. 1990, 56, 2650-2657. [CrossRef] [PubMed]

3. Laub, J.; Henriksen, P.; Brittain, S.M.; Wang, J.; Carmichael, W.W.; Rinehart, K.L. [ADMAdda5]-microcystins inPlanktothrix agardhii strain PH-123 (cyanobacteria)? Importance for monitoring of microcystins in the environment. Environ. Toxicol. 2002, 17, 351-357. [CrossRef]

4. Qin, B.; Zhu, G.; Gao, G.; Zhang, Y.; Li, W.; Paerl, H.W.; Carmichael, W.W. A Drinking Water Crisis in Lake Taihu, China: Linkage to Climatic Variability and Lake Management. Environ. Manag. 2010, 45, 105-112. [CrossRef] [PubMed]

5. Paerl, H.W.; Otten, T.G. Blooms Bite the Hand That Feeds Them. Science 2013, 342, 433-434. [CrossRef] [PubMed]

6. Harke, M.J.; Steffen, M.M.; Gobler, C.J.; Otten, T.G.; Wilhelm, S.; Wood, S.A.; Paerl, H.W. A review of the global ecology, genomics, and biogeography of the toxic cyanobacterium, Microcystis spp. Harmful Algae 2016, 54, 4-20. [CrossRef]

7. Tang, X.; Wu, M.; Yang, W.; Yin, W.; Jin, F.; Ye, M.; Currie, N.; Scholz, M. Ecological Strategy for Eutrophication Control. Water. Air Soil Pollut. 2011, 223, 723-737. [CrossRef]

8. Sun, P.; Esquivel-Elizondo, S.; Zhao, Y.; Wu, Y. Glucose triggers the cytotoxicity of Citrobacter sp. R1 against Microcystis aeruginosa. Sci. Total. Environ. 2017, 603-604, 18-25. [CrossRef]

9. Liu, J.; Yang, C.; Chi, Y.; Wu, D.; Dai, X.; Zhang, X.; Igarashi, Y.; Luo, F. Algicidal characterization and mechanism of Bacillus licheniformis Sp34 against Microcystis aeruginosa in Dianchi Lake. J. Basic Microbiol. 2019, 59, 1112-1124. [CrossRef] [PubMed]

10. Zhang, C.; Massey, I.Y.; Liu, Y.; Huang, F.; Gao, R.; Ding, M.; Xiang, L.; He, C.; Wei, J.; Li, Y.; et al. Identification and characterization of a novel indigenous algicidal bacterium Chryseobacterium species against Microcystis aeruginosa. J. Toxicol. Environ. Health Part A 2019, 82, 845-853. [CrossRef]

11. Zhang, F.; Fan, Y.; Zhang, D.; Chen, S.; Bai, X.; Ma, X.; Xie, Z.; Xu, H. Effect and mechanism of the algicidal bacterium Sulfitobacter porphyrae ZFX1 on the mitigation of harmful algal blooms caused by Prorocentrum donghaiense. Environ. Pollut. 2020, 263, 114475. [CrossRef] [PubMed]

12. Danyang, Z.; Chen, Q.; Zhang, D.; Zhang, H.; Lei, X.; Chen, Z.; Qiuliang, C.; Hong, Y.; Ma, X.; Zheng, W.; et al. The algicidal mechanism of prodigiosin from Hahella sp. KA22 against Microcystis aeruginosa. Sci. Rep. 2017, 7, 1-15. [CrossRef] 
13. Yang, F.; Zhou, Y.; Yin, L.; Zhu, G.; Liang, G.; Pu, Y. Microcystin-Degrading Activity of an Indigenous Bacterial Strain Stenotrophomonas acidaminiphila MC-LTH2 Isolated from Lake Taihu. PLoS ONE 2014, 9, e86216. [CrossRef]

14. Shilo, M. Lysis of Blue-Green Algae by Myxobacter. J. Bacteriol. 1970, 104, 453-461. [CrossRef] [PubMed]

15. Furusawa, G.; Yoshikawa, T.; Yasuda, A.; Sakata, T. Algicidal activity and gliding motility of Saprospira sp. SS98-5. Can. J. Microbiol. 2003, 49, 92-100. [CrossRef]

16. Hou, S.; Shu, W.; Tan, S.; Zhao, L.; Yin, P. Exploration of the antioxidant system and photosynthetic system of a marine algicidal Bacillus and its effect on four harmful algal bloom species. Can. J. Microbiol. 2016, 62, 49-59. [CrossRef]

17. Zhang, X.; Song, T.; Ma, H.; Li, L. Correction: Physiological response of Microcystis aeruginosa to the extracellular substances from an Aeromonas sp. RSC Adv. 2017, 7, 51373. [CrossRef]

18. Shi, S.; Tang, D.; Liu, Y. Effects of an Algicidal Bacterium Pseudomonas mendocina on the Growth and Antioxidant System of Aphanizomenon flos-aquae. Curr. Microbiol. 2009, 59, 107-112. [CrossRef]

19. Wang, H.; Tomasch, J.; Jarek, M.; Wagner-Döbler, I. A dual-species co-cultivation system to study the interactions between Roseobacters and dinoflagellates. Front. Microbiol. 2014, 5, 311. [CrossRef]

20. Gao, X.; Wang, C.; Dai, W.; Ren, S.; Tao, F.; He, X.; Han, G.; Wang, W. Proteomic analysis reveals large amounts of decomposition enzymes and major metabolic pathways involved in algicidal process of Trametes versicolor F21a. Sci. Rep. 2017, 7, 1-10. [CrossRef] [PubMed]

21. de Oliveira, E.J.; Rabinovitch, L.; Monnerat, R.G.; Passos, L.K.J.; Zahner, V. Molecular Characterization of Brevibacillus laterosporus and Its Potential Use in Biological Control. Appl. Environ. Microbiol. 2004, 70, 6657-6664. [CrossRef]

22. Chen, D.; Huang, X.; Li, C.; Zhang, N.; Wei, H.; Zhang, Y. Effects of Brevibacillus laterosporus on microbial community of Microcystis aeruginosa. J. Guangdong Ocean Univ. 2021, 41, 1-9.

23. Zhang, Y.; Chen, D.; Cai, J.; Zhang, N.; Li, F.; Li, C.; Huang, X. Complete Genome Sequence Analysis of Brevibacillus laterosporus Bl-zj Reflects its Potential Algicidal Response. Curr. Microbiol. 2021, 78, 1409-1417. [CrossRef] [PubMed]

24. Rippka, R.; Stanier, R.Y.; Deruelles, J.; Herdman, M.; Waterbury, J.B. Generic Assignments, Strain Histories and Properties of Pure Cultures of Cyanobacteria. Microbiology 1979, 111, 1-61. [CrossRef]

25. Jeffrey, S.; Humphrey, G. New spectrophotometric equations for determining chlorophylls a, b, c1 and c2 in higher plants, algae and natural phytoplankton. Biochem. Physiol. Pflanz. 1975, 167, 191-194. [CrossRef]

26. Wang, T.; Hu, Y.; Zhu, M.; Yin, S. Integrated transcriptome and physiology analysis of Microcystis aeruginosa after exposure to copper sulfate. J. Oceanol. Limnol. 2019, 38, 102-113. [CrossRef]

27. Croce, R.; Amerongen, H.V. Light-harvesting in photosystem I. Photosynth. Res. 2013, 116, 153-166. [CrossRef]

28. Amunts, A.; Drory, O.; Nelson, N. The structure of a plant photosystem I supercomplex at $3.4 \AA$ resolution. Nat. Cell Biol. 2007, 447, 58-63. [CrossRef]

29. Germano, M.; Yakushevska, A.E.; Keegstra, W.; Van Gorkom, H.J.; Dekker, J.P.; Boekema, E.J. Supramolecular organization of photosystem I and light-harvesting complex I in Chlamydomonas reinhardtii. FEBS Lett. 2002, 525, 121-125. [CrossRef]

30. Lushy, A.; Verchovsky, L.; Nechushtai, R. The Stable Assembly of Newly Synthesized PsaE into the Photosystem I Complex Occurring via the Exchange Mechanism Is Facilitated by Electrostatic Interactions. Biochemistry 2002, 41, 11192-11199. [CrossRef] [PubMed]

31. Minai, L.; Fish, A.; Darash-Yahana, M.; Verchovsky, L.; Nechushtai, R. The Assembly of the PsaD Subunit into the Membranal Photosystem I Complex Occurs via an Exchange Mechanism. Biochemistry 2001, 40, 12754-12760. [CrossRef]

32. Hippler, M.; Drepper, F.; Farah, J.; Rochaix, J.-D. Fast electron transfer from cytochrome c6 and plastocyanin to photosystem I of Chlamydomonas reinhardtii requires PsaF. Biochemistry 1997, 36, 6343-6349. [CrossRef]

33. Lunde, C.; Jensen, P.E.; Haldrup, A.; Knoetzel, J.; Scheller, H. The PSI-H subunit of photosystem I is essential for state transitions in plant photosynthesis. Nat. Cell Biol. 2000, 408, 613-615. [CrossRef]

34. Sakata, S.; Mizusawa, N.; Kubota-Kawai, H.; Sakurai, I.; Wada, H. Psb28 is involved in recovery of photosystem II at high temperature in Synechocystis sp. PCC 6803. Biochim. Biophys. Acta (BBA) Bioenerg. 2013, 1827, 50-59. [CrossRef]

35. Cramer, W.A.; Soriano, G.M.; Ponomarev, M.; Huang, D.; Zhang, H.; Martinez, S.E.; Smith, J.L. Some new structural aspects and old controversies concerning the cytochrome b6f complex of oxygenic photosynthesis. Annu. Rev. Plant Physiol. Plant Mol. Biol. 1996, 47, 477-508. [CrossRef] [PubMed]

36. Boekema, E.J.; Braun, H.-P. Supramolecular Structure of the Mitochondrial Oxidative Phosphorylation System. J. Biol. Chem. 2007, 282, 1-4. [CrossRef] [PubMed]

37. Friedrich, T. Complex I: A chimaera of a redox and conformation-driven proton pump? J. Bioenerg. Biomembr. 2001, 33, 169-177. [CrossRef]

38. Cardol, P.; Matagne, R.F.; Remacle, C. Impact of Mutations Affecting ND Mitochondria-encoded Subunits on the Activity and Assembly of Complex I in Chlamydomonas. Implication for the Structural Organization of the Enzyme. J. Mol. Biol. 2002, 319, 1211-1221. [CrossRef]

39. Cardol, P.; Boutaffala, L.; Memmi, S.; Devreese, B.; Matagne, R.F.; Remacle, C. In Chlamydomonas, the loss of ND5 subunit prevents the assembly of whole mitochondrial complex I and leads to the formation of a low abundant $700 \mathrm{kDa}$ subcomplex. Biochim. Biophys. Acta (BBA) Bioenerg. 2008, 1777, 388-396. [CrossRef]

40. Li, Y.; Park, J.-S.; Deng, J.-H.; Bai, Y. Cytochrome c oxidase subunit IV is essential for assembly and respiratory function of the enzyme complex. J. Bioenerg. Biomembr. 2006, 38, 283-291. [CrossRef] 
41. Pierrel, F.; Bestwick, M.L.; Cobine, P.; Khalimonchuk, O.; Cricco, J.; Winge, D.R. Coa1 links the Mss51 post-translational function to Cox1 cofactor insertion in cytochrome c oxidase assembly. EMBO J. 2007, 26, 4335-4346. [CrossRef]

42. Yoshida, M.; Muneyuki, E.; Hisabori, T. ATP synthase-A marvellous rotary engine of the cell. Nat. Rev. Mol. Cell Biol. 2001, 2, 669-677. [CrossRef] [PubMed]

43. Duan, S.; Chen, Q. Transcriptional Analysis of Chlorella Pyrenoidosa Exposed to Bisphenol A. Int. J. Environ. Res. Public Health 2019, 16, 1374. [CrossRef]

44. Guschina, I.A.; Harwood, J.L. Lipids and lipid metabolism in eukaryotic algae. Prog. Lipid Res. 2006, 45, 160-186. [CrossRef] [PubMed]

45. Ruangsomboon, S.; Ganmanee, M.; Choochote, S. Effects of different nitrogen, phosphorus, and iron concentrations and salinity on lipid production in newly isolated strain of the tropical green microalga, Scenedesmus dimorphus KMITL. Environ. Boil. Fishes 2012, 25, 867-874. [CrossRef]

46. Pandit, P.R.; Fulekar, M.H.; Karuna, M.S.L. Effect of salinity stress on growth, lipid productivity, fatty acid composition, and biodiesel properties in Acutodesmus obliquus and Chlorella vulgaris. Environ. Sci. Pollut. Res. 2017, 24, 13437-13451. [CrossRef]

47. Allen, E.E.; Bartlett, D.H. FabF Is Required for Piezoregulation of cis -Vaccenic Acid Levels and Piezophilic Growth of the Deep-Sea Bacterium Photobacterium profundum Strain SS9. J. Bacteriol. 2000, 182, 1264-1271. [CrossRef] [PubMed]

48. Cohen-Gonsaud, M.; Ducasse, S.; Hoh, F.; Zerbib, D.; Labesse, G.; Quemard, A. Crystal Structure of MabA from Mycobacterium tuberculosis, a Reductase involved in Long-chain Fatty Acid Biosynthesis. J. Mol. Biol. 2002, 320, 249-261. [CrossRef]

49. Lai, C.-Y.; Cronan, J.E. $\beta$-Ketoacyl-Acyl Carrier Protein Synthase III (FabH) Is Essential for Bacterial Fatty Acid Synthesis. J. Biol. Chem. 2003, 278, 51494-51503. [CrossRef] [PubMed]

50. Heath, R.J.; Rock, C.O. Roles of the FabA and FabZ $\beta$-Hydroxyacyl-Acyl Carrier Protein Dehydratases in Escherichia coli Fatty Acid Biosynthesis. J. Biol. Chem. 1996, 271, 27795-27801. [CrossRef]

51. Wang, J.; Liu, Q.; Feng, J.; Lv, J.-P.; Xie, S.-L. Effect of high-doses pyrogallol on oxidative damage, transcriptional responses and microcystins synthesis in Microcystis aeruginosa TY001 (Cyanobacteria). Ecotoxicol. Environ. Saf. 2016, 134, 273-279. [CrossRef] [PubMed]

52. Stork, T.; Michel, K.-P.; Pistorius, E.K.; Dietz, K.-J. Bioinformatic analysis of the genomes of the cyanobacteria Synechocystis sp. PCC 6803 and Synechococcus elongatus PCC 7942 for the presence of peroxiredoxins and their transcript regulation under stress. J. Exp. Bot. 2005, 56, 3193-3206. [CrossRef] [PubMed] 